\title{
AISLAMIENTO INSTITUCIONAL DE LOS ENTES DE VIGILANCIA Y CONTROL DE LA PROFESIÓN CONTABLE
}

\section{INSTITUTIONAL ISOLATION OF THE OVERSIGHT AND CONTROL ENTITIES OF THE ACCOUNTING PROFESSION}

\author{
Mario Antonio Ruiz-Vargas ${ }^{1}$ \\ Óscar Saúl Navarro-Morato²
}

\section{RESUMEN}

Este documento presenta los avances del proyecto Redes de corrupción contable, mediante el uso de la información disponible sobre sanciones y sentencias a contadores públicos en Colombia y aplicando técnicas de análisis de redes sociales, se muestra la poca interacción entre las instituciones encargadas de la vigilancia y el control de la profesión contable, así como de la persecución de los delitos financieros. Encontrando que en los casos en los que se evidencia articulación entre entidades están mediados por el carácter público (estatal) del afectado y por el peso mediático del caso, dejando de lado los casos de carácter privado y de poca cuantía pequeñas causas - .

Palabras clave: contador público, corrupción, redes sociales, crimen organizado, ética profesional.

\section{ABSTRACT}

This document presents the advances of the Accounting Corruption Networks project, using available information on sanctions and sentences for public accountants in Colombia and by applying social network analysis techniques, it shows the little interaction between

1 Docente - investigador del Programa de Contaduría Pública de la Universitaria Agustiniana. Doctorando en Administración de Empresas de la Universidad Externado de Colombia.

Correo electrónico: mario.ruiz@uniagustiniana.edu.co Orcid: https://orcid.org/0000-0001-8866-2727

2 Docente - investigador del Programa de Contaduría Pública de la Universitaria Agustiniana. Economista de la Universidad Nacional de Colombia.

Correo electrónico: osnavarrom@unal.edu.co

Orcid: https://orcid.org/0000-0002-0356-7059

Código JEL: D73, D85, L84.

Fecha de recepción: 21/08/2018

Fecha de aceptación: 27/08/2018

DOI: https://doi.org/10.18601/16577175.n22.02 
the institutions in charge of the surveillance and control of the accounting profession, as well as the prosecution of financial crimes. In cases where there is evidence of articulation between entities, this is mediated by the public (state) nature of the affected party and by the weight of the case in the media, leaving aside cases of a private nature and small amounts - crimes of a minor quantity - .

Keywords: accountant, corruption, social network, organized crime, professional ethics.

\section{INTRODUCCIÓN}

Son múltiples las entidades que en el país tienen facultades para aplicar sanciones a los contadores públicos por aquellas conductas y prácticas que van en contra de los presupuestos éticos profesionales o las disposiciones legales. Sin embargo, las conductas antiéticas y corruptas son percibidas por la sociedad colombiana como una práctica común y que tiende a "normalizarse". En el Índice de Percepción de la Corrupción 2016, publicado por Transparencia Internacional, Colombia ocupó el puesto 90 de 176 países. Sin embargo, lo más delicado es que en el entorno de negocios colombiano la existencia de sobornos se ve como algo normal: "[...] la Encuesta Nacional sobre prácticas contra el soborno en Empresas Colombianas, publicado por Transparencia por Colombia y la Universidad Externado, en abril del 2015,90\% de los empresarios del país reconoce que se ofrecen sobornos en el entorno de negocio" (El Tiempo, 2017).
Al abordar el tema de la corrupción en el campo de la ética del profesional de la contaduría pública, es necesario analizar e identificar las relaciones entre profesionales (personas), empresas prestadoras de servicios contables y las instituciones de control. Al observar estás relaciones, se trata de evidenciar la debilidad de las instituciones de control en el dominio del comportamiento y de la ética profesional. Esta debilidad de controles institucionales no es exclusiva del ámbito contable como nos señalan numerosos estudiosos del tema que nos hablan de una crisis ética en Colombia, en la cual la debilidad institucional, cultural y moral son solo parte del problema (Semana, 2017).

La debilidad institucional desde la teoría se muestra altamente relacionada al incremento de la corrupción y a la disminución de la inversión extranjera. Como nos señala Avendaño (2013):

La debilidad institucional se presenta cuando la aplicación de la ley es endeble, prevalece gran discrecionalidad en el empleo de la misma y existen cambios institucionales continuos [...] en un ambiente institucional débil el sistema legal es ineficiente [...] El contexto institucional de un país afecta significativamente el ambiente de negocios y las inversiones. (pp. 367-368)

La literatura sobre crímenes de cuello blanco y ética corporativa es abundante en elementos teóricos y de descripción de modelos de comportamiento de prácticas corruptas, así que al tener el país de manera sostenida índices altos de percepción de corrupción, se observa 
como resultado menores flujos de inversión y, por ende, menor crecimiento económico.

En el presente estudio se identifican aquellas entidades con niveles altos de casos sancionados, el género con mayor cantidad de sanciones y la tipología de las conductas o los delitos sancionados por las entidades, mostrando la (des)articulación de las entidades que cumplen con la función de vigilancia y sanción sobre los profesionales de la contaduría, vigilancia que opera para instituciones y organizaciones en las cuales, en ocasiones, incluso se utilizan recursos de manera improcedente o se producen desfalcos. Si el comportamiento antiético del profesional contable no es sancionado de manera eficaz, se tendrán incentivos para el aumento de las prácticas corruptas, las cuales tendrán incidencia sobre el desempeño económico del país.

\section{Algunos elementos teóricos necesarios para la comprensión de nuestro estudio}

De acuerdo con los Objetivos de Desarrollo Sostenible 2030 de las Naciones Unidas, la corrupción, el soborno, el robo y el fraude fiscal cuestan alrededor de 1260 millones de dólares para los países en desarrollo al año. Esta cantidad de dinero podría ser utilizada para elevar por encima de los 1,25 dólares diarios a las personas que viven con menos de ese dinero durante un mínimo de seis años, por lo que se ha establecido como meta: "Reducir sustancialmente la corrupción y el soborno en todas sus formas" (United Nations
[UN], s. f.). Adicionalmente, para los efectos económicos mencionados antes, como el menor crecimiento económico, la baja IED y el incremento del costo de hacer negocios, existe un costo no computado: un mayor costo de vida para la población en vías en desarrollo (Budak y Vizek, 2015), entre múltiples consecuencias de la corrupción.

La corrupción en las organizaciones cada día está dando un número creciente de víctimas y causando enormes costos. Investigadores internacionales en el tema abordan las causas y consecuencias de la corrupción en las organizaciones y la acción necesaria para reducir los niveles de corrupción en todo el mundo (Anand, Ellstrand, Rajagopalan y Joshi, 2009). Un código de ética no muy bien comunicado puede proliferar corrupción en lugar de reducirla, la forma de manejar el descubrimiento de actos inmorales puede influir en que la organización se enfrente posiblemente a una corrupción en curso en el futuro. De ahí estudios que sugieren formas en que las organizaciones pueden protegerse contra las consecuencias negativas de las prácticas organizativas de rutina. (Joshi, Anand y Henderson, 2007). Otros estudios identifican factores de organización contribuyentes como la institucionalización, la racionalización y la socialización, así como factores personales como locus de control y nivel de desarrollo moral. Estudios que toman un enfoque de interacción de la personalidad, en los que se examinan los papeles que desempeñan los valores y las emociones de los recién llegados en una organización en su aceptación o rechazo al unirse a la corrupción en curso, 
cuando la socialización organizacional los atrae a aceptar elecciones no éticas (Ashforth y Anand, 2003).

La corrupción en Colombia se ha estudiado desde sus bases históricas (Ulloa, 1997; Fajardo, 2002), fundamentos neuropsicológicos (Salcedo-Albarán, Zuleta, Rubio y Beltrán, 2006) costos y crecimiento económicos (Portilla y Gómez, 2002) y la relación con el narcotráfico (Thoumi, 1999), sin embargo, la mayor parte de la investigación en corrupción se ha realizado en el ámbito público, siendo escaso el esfuerzo en la corrupción privada y, en particular, en el fraude corporativo. El estudio del fraude se ha enfocado en el desarrollo de metodologías de auditoría forense y la acción del contador público (Ramírez y ReinaBohórquez, 2013; Leal, Rodríguez y Garzón, 2010), incorporando temas como el gobierno corporativo en las empresas de familia (Baracaldo-Lozano, 2013).

Históricamente el fenómeno de la corrupción es connatural a Colombia, es decir, desde la conquista y la colonia se instauraron estructuras corruptas como parte de la sociedad, fundamentadas en que quienes dirigen envían el mensaje de corrupción, hecho que ha perdurado hasta el día de hoy (Mejía Rivera, 2011). Por esta razón se hace alusión al pensamiento del filósofo chino, Confucio "cuando la dirigencia es corrupta, el pueblo es corrupto", y es que en la esfera ética y moral no vale la teoría, vale el ejemplo.

El escritor y docente Orlando Mejía explica por medio de una anécdota el origen de la corrupción en Colombia:
Cuando llegaban las leyes de india de los reyes de Castilla a estas tierras, los españoles tenían un rito, que consistía en ponerse las leyes sobre la cabeza y decir "lo que el rey quiere, se acata, pero no se obedece" y se hacía lo contrario. A partir de ello, se da explicación a la cultura colombiana, que consiste en la burla sistemática de las leyes, expresada de manera paradójica, es decir, se crean leyes para burlar leyes. (Mejía Rivera, 2011)

Ahora bien, contrastado con la actualidad, se encuentra el caso de los Nule, quienes se vieron involucrados en el escándalo del carrusel de la contratación, donde se resaltan los casos de la calle 26 y la concesión Bogotá - Girardot, este y muchos otros casos son el prototipo de hijos de padres adinerados que aunque incumplen con la ley colombiana siguen teniendo privilegios como la casa por cárcel.

Durante doce años, el país es partícipe de las encuestas que determinan el Índice de Percepción de Corrupción (IPC) en el sector público, realizado por la Organización Transparencia Internacional, donde el parámetro de evaluación es de cero a cien: cero significa que es un país muy corrupto y cien señala que hay menor percepción de corrupción. Ahora bien, durante el tiempo de participación, Colombia se ha quedado en un promedio de 40 , lo que significa que en doce años no ha disminuido el impacto de la corrupción. En el 2016 Colombia ocupó el puesto 90 de 176 países evaluados. Según el informe, el país de la región con mayor percepción es Venezuela, Colombia tiene un puntaje de 37 uni- 
dades y sigue manteniendo la misma puntuación desde el 2014, cuando subió un punto comparado al 2013.

En Colombia se identifican casos de corrupción y fraude del sector privado durante el 2014, como fue la quiebra del Grupo Interbolsa, auditada por la firma Grant Thorton Fast y ABS Auditores; así mismo, se destaca el proceso de la revisora fiscal Luz Adriana Matamba, involucrada en un caso de desfalco a la DIAN relacionado con devoluciones del IVA por operaciones ficticias de comercio exterior, entre otros reportes según González (2014).

Por otro lado, en el sector público se destacan los casos de Coomeva EPS con un desfalco de 146900 millones de pesos, por distorsión de las cuentas de costo, así como en otros gastos que debieron ser asumidos por la entidad y que fueron registrados como costos; el desvío de los recursos parafiscales de la salud, en el que su principal afectado fue el Ministerio de Salud, cuyo desfalco alcanzó 224000 millones de pesos. También el del Grupo Saludcoop, en el que se suspendió por un año a las sociedades Audiogroup SAS y Procesos y Transacciones P\&T Ltda., junto a sus revisores fiscales delegados y contadores de esas compañías; Agro Ingreso Seguro (AIS), con un monto de 12700 millones de pesos, por generación de gastos distintos a difusión, divulgación y socialización de ciencia y tecnología; entre otros casos expuestos en la investigación realizada por Quevedo (2013).

\section{Contabilidad y redes de corrupción $^{3}$}

Neu, Everett, Rahaman y Martínez (2013), en su estudio, ilustran cómo se realiza la contabilidad y por quién, y muestran cómo el "uso hábil" de las prácticas contables y las interacciones sociales en torno a estas prácticas en conjunto permiten la corrupción (p. 505). Estos autores analizaron el programa de patrocinio del Gobierno de Canadá (1994-2003), una iniciativa destinada a fomentar la unidad nacional frente a una amenaza separatista percibida de la provincia de Quebec a mediados de los años noventa. Mientras que se destinaron aproximadamente 322 millones de dólares a este objetivo, entre 13 millones de dólares y 50 millones de dólares fueron desviados a las cuentas de partidos políticos, administradores de programas, así como a sus familias, amigos y compañeros de trabajo (la cifra exacta es desconocida) (Neu, Everett, Rahaman y Martínez, 2013,p. 506).

Este estudio hace dos contribuciones principales a nuestra comprensión de la contabilidad. En primer lugar, ilustra cómo la contabilidad simultáneamente limita y permite la corrupción en los países capitalistas avanzados. En segundo lugar, el estudio destaca la centralidad de la contabilidad dentro de las redes criminales organizadas. Las alianzas criminales organizadas, que implican la corrupción (Di Nicola, 2003, 2006), el lavado de dinero (Beare y Schneider, 2007, Mitchell, Sikka y Willmott, 1998, 
Reuter y Truman, 2004) y el fraude corporativo sistémico (Baker y Hayes, 2004), a veces se basan en redes que se construyen explícitamente en torno a la realización de un conjunto específico de transacciones contables (Neu, Everett, Rahaman y Martínez, 2013, p. 507).

Además, este estudio examina cómo la contabilidad hace posible la corrupción dentro de un entorno de mercado de influencia. El análisis demuestra que si bien la lucha contra la corrupción está basada en la contabilidad, las barreras intentan limitar y restringir la forma en que se compra y se vende la influencia política, el ejercicio colaborativo de la discreción y el uso colaborativo de estrategias contables implican modificar y aumentar el flujo de recursos gubernamentales a los actores empresariales $\mathrm{y}$, posteriormente, repartir una porción de estos ingresos a los actores políticos involucrados (Neu, Everett, Rahaman y Martínez, 2013, p. 521).

Estas prácticas contables están informadas y configuradas por el habitus del campo, su distribución de capital y el posicionamiento espacial de influencias sociales; convirtiendo a la contabilidad en el eje entorno al cual se organiza la red corrupta, mediante la utilización de patrones particulares de prácticas contables y las interacciones sociales alrededor de estas prácticas. Así, al lograr incorporar tales prácticas en el habitus de la red $-\mathrm{y}$, por tanto, en los repertorios de sus participantes-, el hábil uso de la contabilidad es valorada como una forma de capital simbólico. En otras palabras, las prácticas contables llegan a organizar la emergente red corrupta (Neu, Everett, Rahaman y Martínez, 2013, p. 521).

En estos contextos, la contabilidad es algo más que simplemente una barrera para las prácticas corruptas, contrario a lo que se había asumido en investigaciones previas a cerca de la corrupción, y es a su vez, más que una estrategia como se expone en la literatura sobre contabilidad creativa (Griffiths, 1986, 1995, de la Torre, 2009). Con el riesgo de exageración, los autores sugieren que las prácticas contables son la columna vertebral de este tipo de red corrupta, dando forma a las prácticas y las interacciones al interior de la red y la estructura misma de la red (Neu, Everett, Rahaman y Martínez, 2013, p. 521). El estudio de Neu et al. (2013) se ha centrado en la forma en que la contabilidad organiza la corrupción, pero como los propios autores lo sugieren es necesario un trabajo adicional sobre las prácticas sociales de prevención, detección, investigación y enjuiciamiento de las actividades delictivas de cuello blanco. La ironía, por supuesto, es que mientras estos ajustes son difíciles de estudiar, también son los mismos ajustes en los que es más importante entender cómo funcionan la contabilidad y la auditoría.

Johnston (2015), en su investigación se basa en un marco de gobierno para considerar la lucha global contra la corrupción y el papel que desempeña la contabilidad en esa lucha. Específicamente, examina dos modos de pensamiento o "mentalidades" que contrastan en el campo de la anticorrupción y la forma en que forman, imaginan y constituyen la práctica cultural y tec- 
nológica de la contabilidad. La primera mentalidad, la mentalidad ortodoxa, es evidente en los discursos programáticos y en las investigaciones académicas de las principales organizaciones anticorrupción (especialmente, el Banco Mundial, las Naciones Unidas, el Fondo Monetario Internacional [FMI], la Organización para la Cooperación y el Desarrollo Económicos [OCDE] y Transparencia Internacional). Esta mentalidad nos ha hecho imaginar la participación de la contabilidad en este campo como parte de una causa poco problemática, si no noble. La segunda mentalidad radical se basa en comentarios de raza, género y clase, y nos hace ver la participación de la contabilidad en términos más ambivalentes: como potencial habilitador, pero también un potencial limitador de la responsabilidad económica y el logro del sueño liberal republicano (p. 97).

Everett, Neu y Rahaman (2007) exploran el problema de la corrupción y discute este fenómeno desde dos grandes perspectivas, la ortodoxa y la radical. La primera señala que el papel que desempeña la contabilidad en la lucha contra la corrupción es poco problemático en este campo, aunque reconoce una serie de dificultades, esta perspectiva considera que la contabilidad es esencialmente una parte de una "causa noble". La contabilidad, desde este punto de vista, realmente solo tiene que "hacer más" si se quiere ganar la lucha. En contraste, una mentalidad más marginal y fragmentada, a la que denominamos "radical", apunta a una función más ambivalente para la práctica cultural de la contabilidad (p. 535).
Además, argumenta que la contabilidad, como práctica performativa e intermediaria entre el proyecto teórico-analítico de la lucha contra la corrupción y su manifestación práctica programática, tiene el potencial de evitar una forma alternativa de rendición de cuentas económicas, más adaptada a las necesidades de las víctimas de la corrupción. Esto amplía la "función de la información" de la contabilidad (Kimbro, 2002) más allá de una visión ortodoxa. Sostiene y argumenta que se reproduce un dualismo falso y peligroso (economía/ sociedad). Reconoce que habrá muchos problemas para organizaciones como la Federación Internacional de Contadores (IFAC) y la Organización Internacional de Entidades Fiscalizadoras Superiores (INTOSAI) en el diseño e implementación de iniciativas que respondan mejor a las necesidades de las verdaderas víctimas de la corrupción, pero estos problemas no serán abordados si no hay primero un reconocimiento y conciencia del hecho de que la visión actual del campo para la contabilidad es bastante limitante (Everett, Neu y Rahaman, 2007, p. 535).

Lo aquí planteado hasta ahora tiene por objeto abordar la estructura institucional de vigilancia y control de la profesión contable, en el marco de un problema generalizado en la sociedad global como la corrupción, utilizando como herramienta el análisis de redes sociales y así entrever posibles vacíos o desarticulaciones institucionales.

\section{MATERIALES Y MÉTODOS}

Mediante el análisis de redes se busca identificar la densidad de los delitos/ 
conductas sancionadas por los diferentes organismos y la interconexión entre entidades mediante la identificación de profesionales que hayan sido sancionados por más de una entidad por un mismo caso/evento ${ }^{4}$.

Los datos utilizados en este avance de investigación provienen de las publicaciones realizadas por las entidades de interés en que en este estudio son: Junta Central de Contadores (JCC), Fiscalía General de la Nación (FGN), Superintendencia Financiera (SFN) y la Dirección de Impuestos y Aduanas Nacionales (DIAN); relacionadas con sanciones a profesionales de contaduría pública, de acuerdo con las competencias legales que tiene cada una de estas entidades.

Se cuenta hasta el momento una base de datos con 1004 registros que contiene información de casos desde 2004 hasta el 2016; dichos registros se refieren a resoluciones y procesos que se han cerrado en dichos órganos de control, contra profesionales de contaduría pública, ya sea en cuanto a la comisión de acciones en contra de la ética profesional (aspectos disciplinarios) o de delitos (aspectos ilícitos).

Por su parte, el análisis de redes sociales se basa en la noción de una "red social" entendida como un conjunto finito de actores y las relaciones que los vinculan, asimismo, las redes sociales son consideradas estructuras sociales en las que se producen procesos de comunicación y transacción entre personas y organizaciones. Es un paradigma de las ciencias sociales que busca generar explicaciones sobre los fenómenos sociales concentrando su atención en los patrones y las estructuras relacionales que componen sistemas de relacionamiento e intercambio.

La utilización del Análisis de Redes Sociales aplicado al fenómeno criminal es de vieja data, y se remonta a los inicios de este paradigma en la primera mitad del siglo pasado, como lo evidencian los trabajos reseñados por McIllwain (1999); Carrington (2011); Bouchard y Malm (2016) y Gravel y Tita (2017), entre otros.

Esta primera mirada al sistema de instituciones que interactúan bajo el marco legal colombiano, y que cuentan con la capacidad de sancionar a los profesionales de la contaduría pública, busca establecer la centralidad de dichas interacciones, el peso de cada entidad y cómo se refleja la importancia de estas instituciones que deben luchar contra la corrupción; y en este caso particular, dado el peso que tienen los contadores públicos en la comisión de actos antiéticos y de delitos relacionados con el patrimonio económico privado y público, identificar cuál es el real actuar de estos en la identificación y persecución de delitos cometidos por estos profesionales.

\section{Resultados y discusión}

Inicialmente, y como se muestra en el gráfico 1 , se tiene como principal ente sancionador a la JCC siendo la entidad con mayor número de profesionales 


\section{Gráfico 1.}

Registros por entes sancionadores (2004-2016)

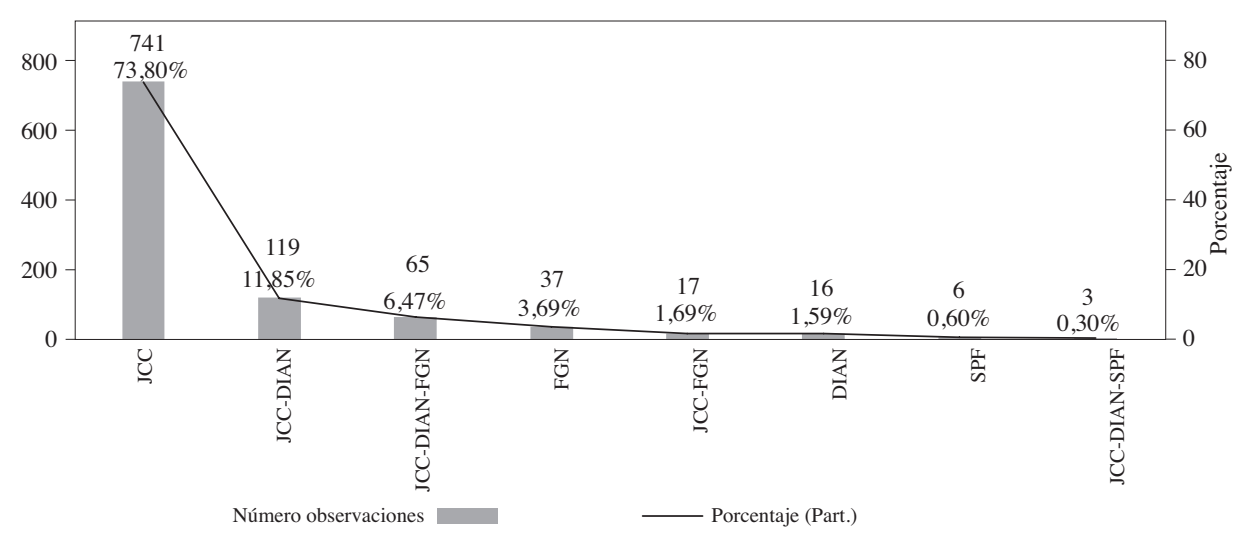

Fuente: elaboración propia, con base en datos recopilados por las cuatro entidades mencionadas.

contables sancionados, con un $73,80 \%$ de los 1004 registros, seguida de la FGN, con apenas un $3,69 \%$, la DIAN solamente ha sancionado dieciséis profesionales, es decir, el $1,59 \%$, y, finalmente, la SPF apenas ha sancionado a seis contadores $(0,6 \%)$.

En cuanto a registros que muestran interacción entre organismos sobre un mismo individuo, se observa que 119 registros exponen situaciones en las que profesionales sancionados por conductas antiéticas por parte de la JCC, también lo fueron por parte de la DIAN, lo que da un indicio de que las conductas cometidas por estos individuos están cercanamente relacionados con temas tributarios, a su vez, existen otros 65 registros en los cuales no solo la JCC y la DIAN intervinieron, lo hizo también la FGN, lo cual indica que no solo se cometieron faltas disciplinarias, sino que además, dichas faltas a la ética del profesional contable, tenían como objetivo la comisión de delitos - penalizados por la legislación colombiana - y en los cuales la FGN pudo recabar pruebas de dichas conductas. Finalmente, se cuenta con diecisiete registros para los cuales se dan acciones tanto de la JCC como de la FGN y solamente para tres registros se tiene una interacción entre la JCC, la DIAN y la SFN.

Al presentar esta información en un grafo en el cual se tiene que los registros se interrelacionan a través de la entidad u organismo sancionador, se obtienen una red como la que se presenta en el gráfico 2 .

Lo que se busca con el cruce de información es evidenciar la (in)eficiencia del sistema de control y sanción del contador público en el país y las tendencias de comportamiento ético de los profesionales sancionados durante el periodo de análisis.

Por tanto, los casos de interés son aquellos sancionados por más de una 
Gráfico 2.

Contadores públicos sancionados de acuerdo con el ente sancionador (2004-2016)

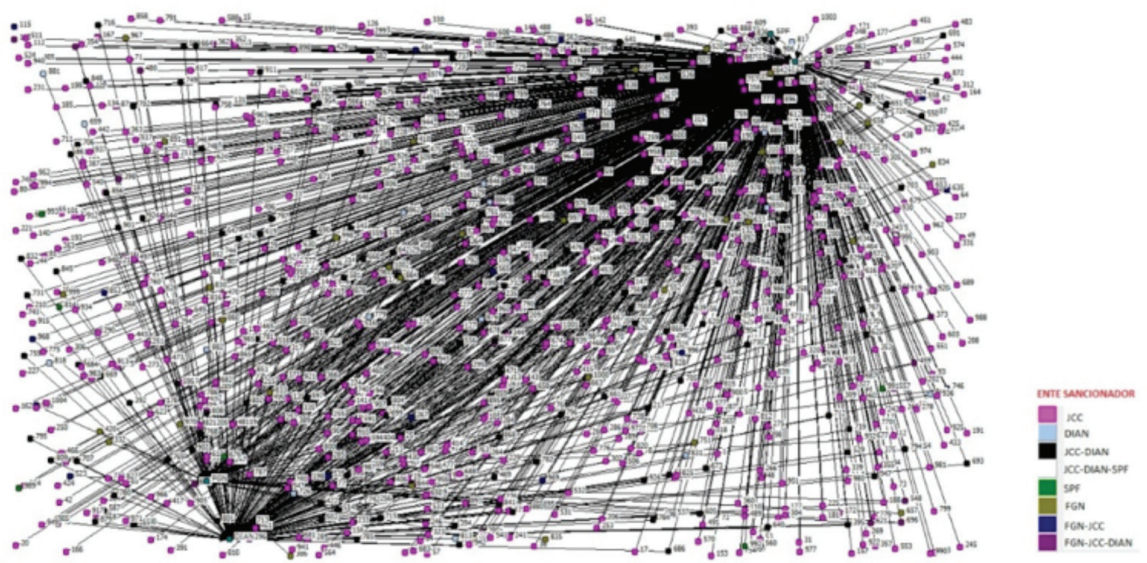

Fuente: elaboración propia, con base en datos recopilados de las cuatro entidades mencionadas.

entidad, con el fin de identificar la conducta o delito sancionado/penalizado y la "sinergia" entre las entidades. Del total de registros, apenas 204 muestran alguna interacción entre al menos, dos de las entidades que se analizan, - es decir, apenas el 20,32\% de estos-; $\mathrm{y}$, por ende, los restantes ochocientos registros se dan como acciones aislada o unilaterales por parte de uno de los organismos referidos y en los que la persecución a las conductas de tipo penal - verificado por la participación de la FGN-, apenas si llega al 9,37\%

Como ya se ha mencionado, los registros con sanciones emanadas tanto de la JCC, como de la FGN en conjunto con la DIAN apenas llegan a los 65, que representan apenas el $6,47 \%$ del total de registros. El gráfico 3 muestra dichos casos, distinguiendo por el cargo ostentado por el implicado y por el tipo de sanción recibida, - al momento de consultar las bases de datos disponibles- .

En el gráfico 4 se muestra la subred configurada por los registros sobre sanciones en los que intervienen la JCC, la DIAN y la SFN, al hacer la búsqueda de la descripción del caso, se encuentra que estos registros hacen referencia al contador y al revisor fiscal de la firma Grant Thornton Fast \& ABS auditores Ltda., que fue la encargada de la revisoría fiscal de Interbolsa, conocido escándalo de corrupción que afecto a cientos de inversionistas.

La prensa registró el hecho, como se aprecia en la nota de El Tiempo - editor de Economía (2015):

La decisión del Tribunal [Disciplinario de la Junta Central de Contadores] involucra también a los contadores públicos 


\section{Gráfico 3.}

Contadores públicos sancionados simultáneamente por JCC, FGN y DIAN (2004-2016)

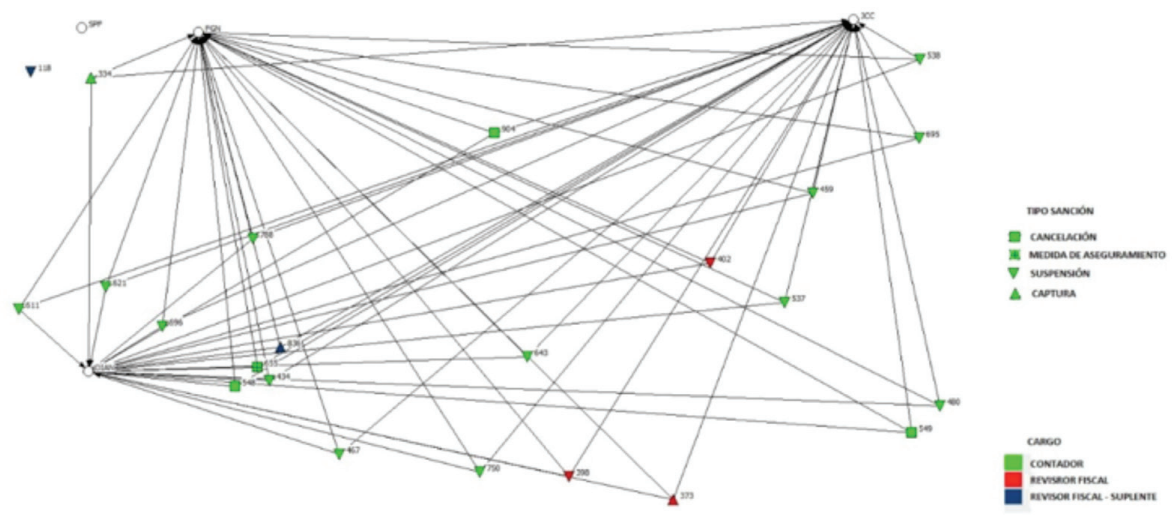

Fuente: elaboración propia, con base en datos recopilados de las cuatro entidades mencionadas.

Gráfico 4.

Contadores públicos sancionados simultáneamente por JCC, DIAN y SFN (2004-2016)

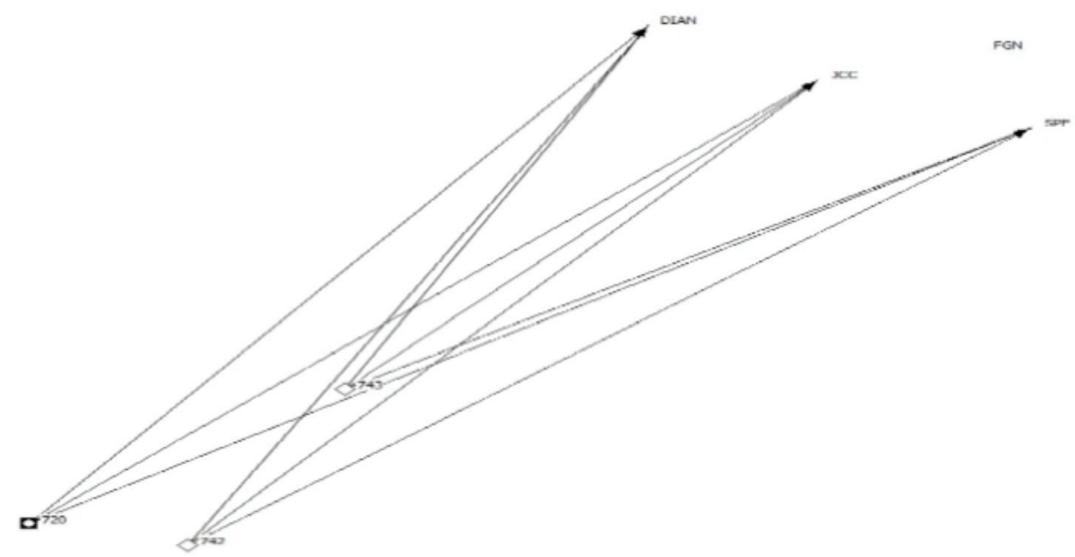

Fuente: elaboración propia, con base en datos recopilados de las cuatro entidades mencionadas.

U.C. L. (revisora fiscal de Interbolsa) y C.A. P. (contador de la misma), a quienes les suspenden la tarjeta profesional por 12 meses por 'haber vulnerado el estatuto ético de la profesión'.
La instancia disciplinaria de los contadores señala que los sancionados 'no se pronunciaron en su debida oportunidad, ni ejercieron las medidas de control pertinentes frente a las irregularidades 
Gráfico 5.

Contadores públicos sancionados por la JCC (2012-2016)

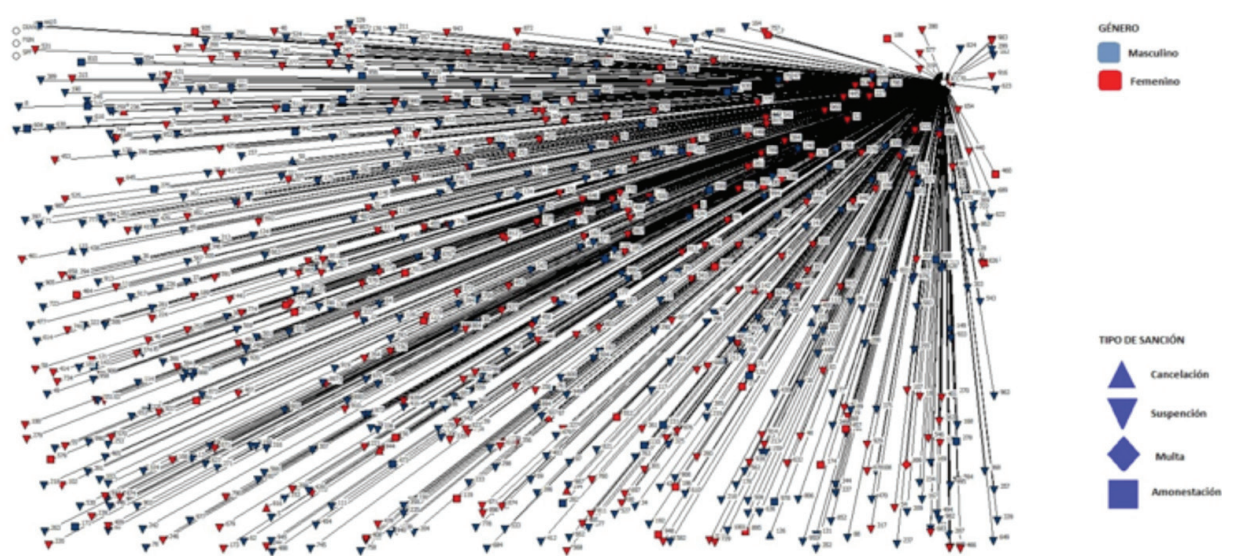

Fuente: elaboración propia, con base en datos recopilados de las cuatro entidades mencionadas.

contables para el periodo de 2012, frente a situaciones tales como el riesgo de iliquidez que representaba para la sociedad la concesión de préstamos particulares, pertenecientes al Grupo Interbolsa, que además eran otorgados sin garantías reales'.

Como lo muestra el gráfico 5, el principal ente que se pronuncia sobre la conducta ética del contador es la JCC para la ventana de tiempo del 2012 a 2016, la tendencia en cuanto a la actuación del Tribunal Disciplinario es la de un aumento paulatino en el número de sanciones impuestas hasta el 2014, luego del cual se presenta un descenso en los casos fallados por dicha instancia, una posible explicación podría ser el impacto que durante el periodo 2010-2014 tuvieron casos como: DMG, desfalcos a la DIAN e Interbolsa.
El tipo de sanción más utilizado por la JCC es la suspensión de la tarjeta profesional - exactamente 841 registros con un $90,24 \%$ para el periodo $2007-$ 2016; mientras que solamente cuatro registros $-0,43 \%$ - corresponden a multas y 36 registros corresponden a cancelaciones de dicho documento $-3,86 \%-$, con picos en el 2013 y el 2015 (ver gráfico 6)

De los registros analizados, tan solo 37 corresponden a casos de contadores sancionados por la FNG (ver figura 7), de manera curiosa - por decir lo menos-, son casos asociados a profesionales reincidentes en sanciones y procesos judiciales en curso. Al depurar los registros hasta ahora obtenidos, los 1004 registros hacen referencia a 826 personas, lo que implica la reincidencia de algunos profesionales y diferentes 
Gráfico 6.

Participación \% por tipo de sanción impuesta por la JCC (2007-2016)

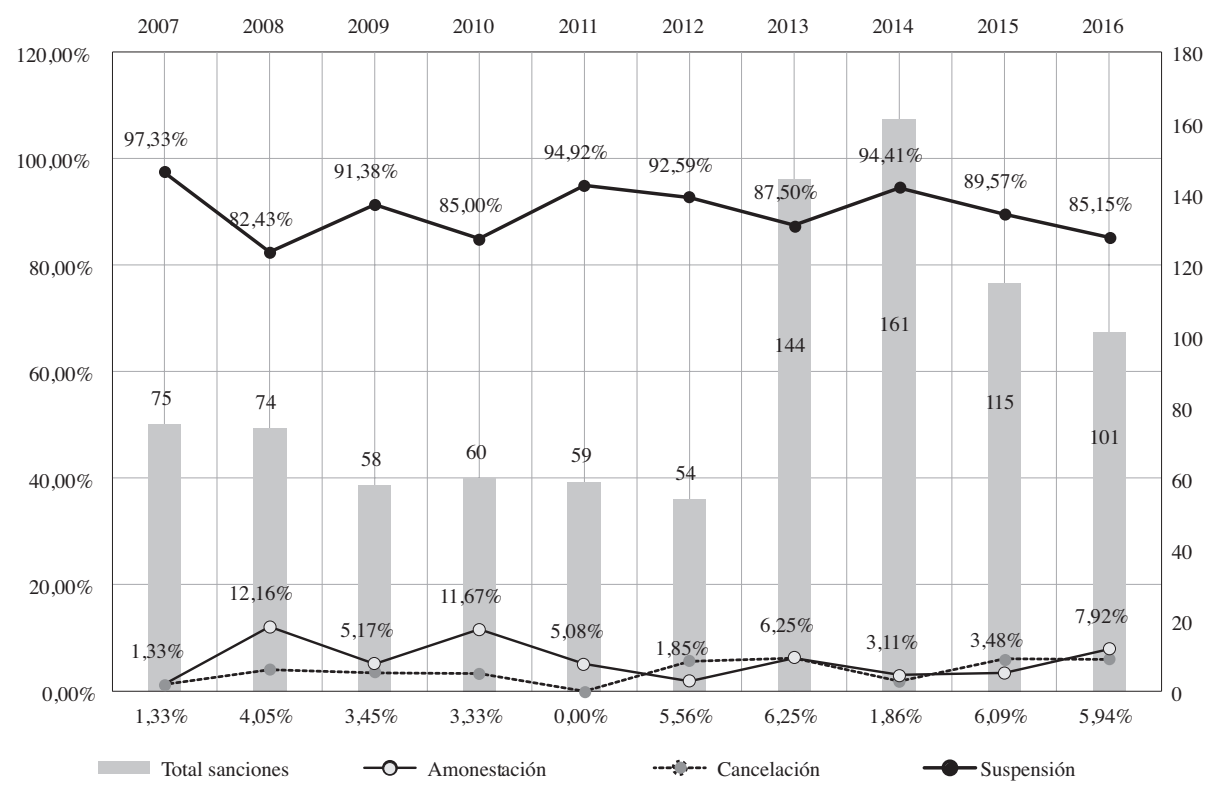

Fuente: elaboración propia, con base en datos recopilados de la JCC.

resoluciones emitidas por los entes de control -en diferentes momentos de tiempo- para algunos profesionales ${ }^{5}$; específicamente, se encuentran diez profesionales con cuatro o más registros de sanción por parte de alguna entidad de control.

El primer lugar es ocupado por una profesional relacionada con un caso de defraudación al Estado mediante la creación de empresas de papel, que realizaban operaciones ficticias de importación y exportación de "chatarra", y la solicitud de devolución del IVA. Un extracto de la nota publicada por la DIAN (2013) en el periódico El Espectador (2013) se muestra a continuación:
[...] Según información de la Subdirección de Registro Aduanero, estas comercializadoras internacionales no reportan declaraciones de exportación. Tampoco presentaron el informe anual de compras y exportaciones de los años 2008 y 2009. 6.- Llama la atención, al despacho, que cinco de las sociedades relacionadas como proveedores fueron creadas en la misma fecha (Marzo 19 de 2009), más aún, las señoras A. B. M. y L.P. H. A. que figuran como contadores o revisoras fiscales de CHATARRA UNIVERSAL MONTERIA S.A.S, METAL SANTANDER S.A.S, CHATARRA DEL NORTE KYM S.A.S., METALES OCHOA S.A.S., CHATARRA PUERTO S.A.S Y C.I. PACIFIC METAL INTERNACIONAL S.A. 


\section{Gráfico 7.}

Contadores públicos sancionados por la FGN (2014-2016)

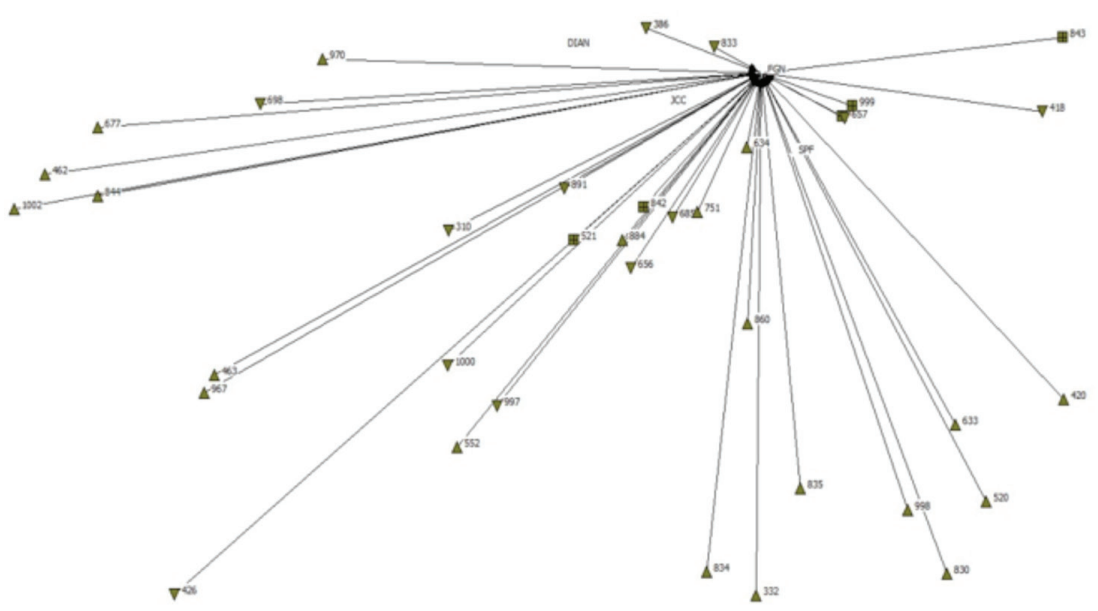

Fuente: elaboración propia, con base en datos recopilados de la FGN.

están sindicadas del desfalco al Estado por la devolución de IVA, originado en ficticios procesos de exportación [...].

La profesional en cuestión tiene once registros sancionatorios por parte de la JCC y tres de la DIAN, además, parte de una investigación que adelantó la FGN y que en el 2012 arrojó el desmantelamiento de una organización delictiva que:

[...] desfalcó a la Dian en Medellín, por un monto aproximado a los $\$ 100$ mil millones de pesos mediante empresas ficticias que cobraban la devolución del Impuesto al Valor Agregado, IVA, con documentación falsa.

Dicha red, de acuerdo con la investigación, habría realizado las defraudaciones simulaban total o parcialmente hechos económicos para lo cual utilizaba fac- turas falsas, y certificaciones de exportaciones inexistentes con la anuencia de funcionarios de esa seccional. En las diligencias de allanamiento fueron capturadas 11 personas, entre ellas la revisora fiscal J. V.S. S., quien también hace parte del grupo que desfalcó a la Dian en Bogotá. (Unidad Nacional de Fiscalías, 2012)

En dicho caso, el proceso que la vincula al desfalco de la DIAN ya da cuenta del carácter nacional de las actuaciones de estos profesionales. Los otros nueve implicados en el proceso llevado por la fiscalía y que son profesionales contables, también muestran reincidencia en la comisión de actos antiéticos, sancionados por la JCC.

Antes de finalizar, es de resaltar el hecho de que tanto en el ámbito ético como en el penal, las sanciones al 
Gráfico 8.

Distribución \% de participación por género en sanciones (2007-2016)

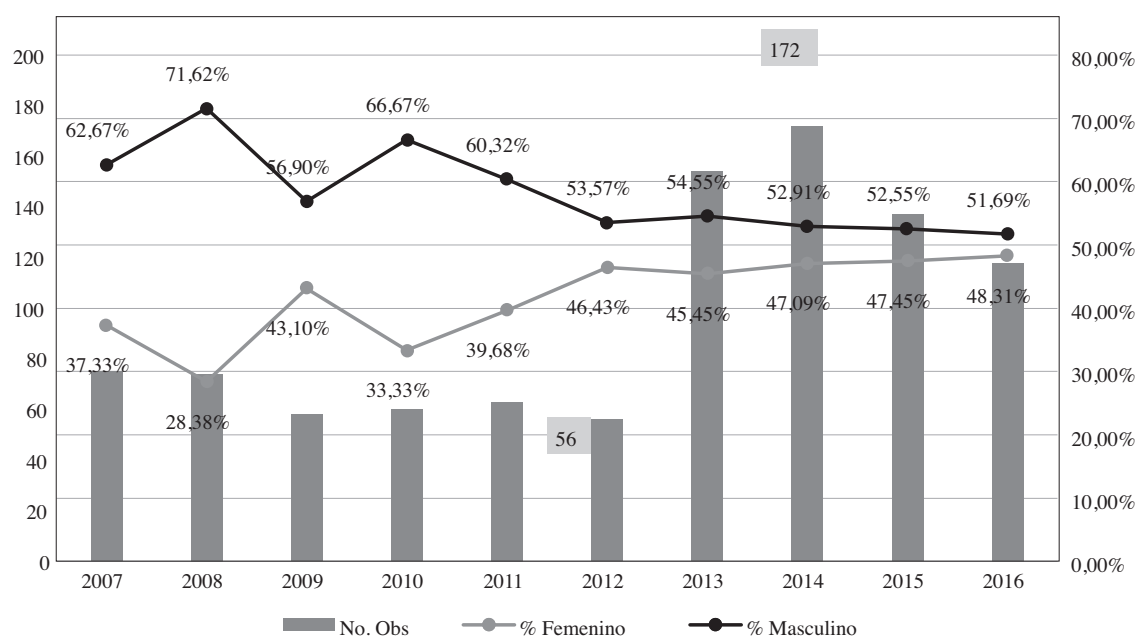

Fuente: elaboración propia, con base en datos recopilados de las cuatro entidades mencionadas.

Gráfico 9.

Número de registros de profesionales investigados por la FGN. Por género (2010-2016)

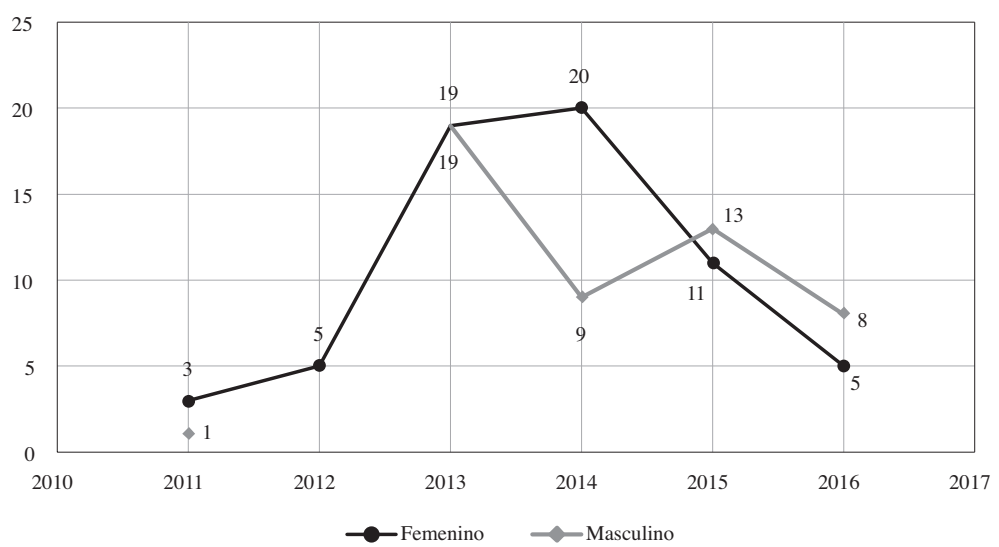

Fuente: elaboración propia, con base en datos recopilados de las cuatro entidades mencionadas.

género femenino en el transcurso del tiempo se equiparan con el del género masculino, en algunos casos las cifras superan al segundo (ver gráficos $8 \mathrm{y}$ 9). Como posible explicación a este fenómeno, que debería ser analizado con mayor interés, puede ser el com- portamiento del género de los profesionales graduados, dado que son más las mujeres que los hombres que obtienen el título de contador público, además, del posicionamiento de la mujer en cargos de mayor responsabilidad en las organizaciones. 


\section{CONCLUSIONES}

$\mathrm{Al}$ tener en cuenta las relaciones entre las instituciones de control y los profesionales sancionados, el panorama permite evidenciar cierto aislamiento, que lleva a la debilidad de las instituciones de control y puede desembocar en una desarticulación institucional en contra de las políticas anticorrupción, lo cual puede ser tomado por los profesionales de la contaduría pública como un incentivo para la comisión de prácticas corruptas.

Las interacciones entre las instituciones se enmarcan, en principio, en la investigación y sanción de los casos de mayor "relevancia" mediática, sin avanzar significativamente en aquellas prácticas desarrolladas en entes u organizaciones a pequeña y mediana escala; como se mostró en los comentarios a las gráficos 3 y 7 , lo disciplinario y lo penal se integran a la hora de perseguir grandes desfalcos al erario o escándalos que afectan a ciertas capaz de la sociedad. En otras palabras, se traducen en que si el comportamiento antiético del profesional contable no es sancionado de manera eficaz, se tendrán incentivos para el aumento de las prácticas corruptas, las cuales tendrán incidencia sobre el desempeño económico del país.

Hace parte de la línea de trabajo del proyecto de investigación que se está desarrollando, la posibilidad de demostrar no solo la debilidad, sino también la desarticulación de las instituciones de vigilancia control de la profesión contable, en el marco de las políticas locales y globales de la lucha contra la corrupción.

\section{REFERENCIAS BIBLIOGRÁFICAS}

Anand, V., Ellstrand, A., Rajagopalan, A. y Joshi, M. (2009). 10 Organizational responses to allegations of corporate corruption. En R. Burke y C. Cooper (eds.), Research companion to corruption in organizations (pp. 217-230). Cheltenham: Edward Elgar Publishing.

Ashforth, B.E. y Anand, V. (2003). The normalization of corruption in organizations. Research in organizational behavior, 25, 1-52.

Avendaño Miranda, L. (2013). Las ventajas de la debilidad institucional: reflexiones sobre la IED china en África Subsahariana y América Latina. Foro Internacional, 53(2), 363-396.

Baker, R. y Hayes, R. (2004). Reflecting form over substance: The case of Enron Corp. Critical Perspectives on Accounting, 15(6-7), 767-785.

Baracaldo-Lozano, N. A. (2013). Diagnóstico de gobierno corporativo como mecanismo en la prevención del fraude en empresas familiares. Aplicación de método de casos. Cuadernos de Contabilidad, 14(35), 581-615.

Beare, M. y Schneider, S. (2007). Money laundering in Canada: Chasing dirty and dangerous dollars. Toronto: University of Toronto Press.

Bouchard, M. y Malm, A. (2016). Social Network Analysis and Its Contribution to Research on Crime and Criminal Justice. Vol. 1. Oxford University Press.http://doi.org/10.1093/ oxfordhb/9780199935383.013.21

Budak, J., y Vizek, M. (2015). The Corruption Mark-up: is corruption cost 
incorporated in the prices of goods and services in emerging and developed countries? Post-Communist Economist, 27(2), 247-255

Carrington, P. J. (2011). Crime and Social Network Analysis. In J. Scott y P. J. Carrington (eds.), The SAGE Handbook of Social Network Analysis (236-255). Croydon: SAGE Publications.

De la Torre, I. (2009). Creative accounting exposed. Nueva York: Palgrave Macmillan.

Di Nicola, A. (2006). La Criminalita Organizzata. Milán: Franco Angeli.

Dirección de Impuestos y Aduanas Nacionales (DIAN) (2013). Resolución sanción declarando proveedor ficticio. Dirección Seccional de Impuestos y Aduanas de Villavicencio. En El Espectador (2013).

El Espectador. (22 de julio del 2013). Dirección de Impuestos y Aduanas Nacionales. Publicación de resoluciones. Recuperado de http://static. elespectador.com/archivos/2013/ 07/778779a2e5fe7ff684e301f98c af9461.pdf

El Tiempo - Editor de Economía. (2015). Quitan licencia a firma de revisoría fiscal de Interbolsa. Recuperado de http://www.eltiempo.com/archivo/ documento/CMS-16263697

El Tiempo (25 de enero del 2017). Colombia, rajado en 2016 en percepción de transparencia. (Redacción El Tiempo, editor). Recuperado de http:// www.eltiempo.com/justicia/cortes/ percepcion-de-corrupcion-en-colombia-empeoro-en-2016-29242

Everett, J., Neu, D. y Rahaman, A. S. (2007). Accounting and the global fight against corruption. Accounting,
Organizations and Society, 32(6), 513-542.

Fajardo, L. (2002). La corrupción heredada: pasado colonial, sistema legal y desarrollo económico en Colombia. Revista de Estudios Sociales, (12), 22-30.

Gravel, J. y Tita, G. E. (2017). Network Perspectives on Crime. In Oxford Research Encyclopedia of Criminology and Criminal Justice. Vol. 1. Oxford University Press. http://doi.org/10.1093/acrefore/9780190264079.013.251

González, P. F. (16 de mayo del 2014). Contadores, en la mira por grandes escándalos del país. El Tiempo. Recuperado de http://www.eltiempo. com/archivo/documento/CMS 13994576

Griffiths, I. (1986). Creative accounting: How to make your profits what you want them to be. Londres: Routledge.

Griffiths, I. (1995). New creative accounting. Nueva York: Palgrave MacMillan.

Johnston, M. (2015). Making transparency real? Accounting and popular participation in corruption control. Critical Perspectives on Accounting, 28, 97-101.

Joshi, M., Anand, V. y Henderson, K. (2007). The role of organizational practices and routines in facilitating normalized corruption. En J. Langan-Fox, C. L. Cooper y R. J. Klimoski (eds.), Research companion to the dysfunctional workplace: $\mathrm{Ma}$ nagement challenges and symptoms (pp. 235-251). Cheltenham: Edward Elgar Publishing. https://doi. org/10.4337/9781847207081.00022 
Leal, L., Rodríguez, E. y González, R. (2010). Auditoría forense: los procedimientos del contador público en la investigación del fraude corporativo. Gestión y Sociedad, 3(2), 141-160.

McIllwain, J. S. (1999). Organized crime: A social network approach. Crime, Law and Social Change, 32(4), 301-323. http://doi. org/10.1023/A:1008354713842

Mejía Rivera, O. (18 de octubre del 2011). El origen de la corrupción en Colombia. El Radar. (B. Gómez, entrevistador) Caracol Televisión. Recuperado de https://noticias. caracoltv.com/el-radar/el-origen-dela-corrupcion-en-colombia

Mitchell, A., Sikka, P. y Willmott, H. (1998). Sweeping it under the carpet: The role of accountancy firms in money laundering. Accounting, Organizations and Society, 23(5/6), 589-607.

Neu, D., Everett, J., Rahaman, A. S. y Martínez, D. (2013). Accounting and networks of corruption. Accounting, Organizations and Society, 38(6), 505-524.

Portilla, K. G. y Gómez, S. G. (2002). El impacto de la corrupción sobre el crecimiento económico colombiano, 1990-1999. Lecturas de Economía, (57), 49-86.

Quevedo, N. (20 de septiembre del 2013). El top 10 de los casos de corrupción "ad-portas" de fallo. El Espectador. Recuperado de http:// www.elespectador.com/noticias/ investigacion/el-top-10-de-loscasos-de-corrupcion-ad-portas-defallo-articulo-447582
Ramírez, M. y Reina Bohórquez, J. (2013). Metodología y desarrollo de la auditoría forense en la detección del fraude contable en Colombia. Cuadernos de Administración, 29(50), 177-195.

Reuter, P. y Truman, E. (2004). Chasing dirty money: The fight against money laundering. Washington: Institute for International Economics.

Salcedo-Albarán, E., Zuleta, M. M., Rubio, M. y de León-Beltrán, I. (2006). Neuronas espejo, teoría de la mente y corrupción. Neuropsicología para prevenir la corrupción (n. $\left.{ }^{\circ} 003272\right)$. Grupo método.

Semana (7 de agosto del 2017). ¿En qué momento se acabó la ética en Colombia? Recuperado de http:// www.semana.com/nacion/articulo/ seis-academicos-reflexionan-sobrela-crisis-etica/531795

Thoumi, F. (1999). La relación entre corrupción y narcotráfico: un análisis general y algunas referencias a Colombia. Revista de Economía del Rosario, 2(1), 11-33.

Ulloa, F. C. (1997). La corrupción en Colombia. Bogotá: TM Editores.

Unidad Nacional de Fiscalías. (2012). Fiscalía descubre millonario desfalco a la DIAN en Medellín. Recuperado de https://www.fiscalia.gov.co/ colombia/noticias/fiscalia-descubre-millonario-desfalco-a-la-dianen-medellin/

United Nations (UN) (s. f.). Objetivos de desarrollo sostenible. 17 objetivos para transformar nuestro mundo. UN. Recuperado de http://www. un.org/sustainabledevelopment/es/ peace-justice/ 